\title{
Numerical quantification model and experiment of external force on roller hemming of curved edge aluminium alloy with adhesive
}

Jianjun Li

Tongji University

Wenfeng Zhu ( $\nabla$ zhuwenfeng@tongji.edu.cn )

Tongji University https://orcid.org/0000-0002-9096-6751

Yuanhui Li

Tongji University

\section{Original Article}

Keywords: External force, Forming, Adhesive, Simulation, Aluminum alloy sheet, Manufacturing quality

Posted Date: August 17th, 2021

DOI: https://doi.org/10.21203/rs.3.rs-806101/v1

License: (c) (i) This work is licensed under a Creative Commons Attribution 4.0 International License.

Read Full License

Version of Record: A version of this preprint was published at Chinese Journal of Mechanical Engineering on March 2nd, 2022. See the published version at https://doi.org/10.1186/s10033-022-00685-5. 


\section{Title page}

\section{Numerical quantification model and experiment of external force on roller hemming of curved edge aluminium alloy with adhesive}

Jian-Jun Li, born in 1984, is currently a PhD candidate at School of Mechanical Engineering, Tongji University, China. He received his master degree from Shandong University, China, in 2011. His research interests include lightweight manufacturing of car body, material forming technology, mechanical behavior of materials and structures.

Tel: +86 18953185993; E-mail: lijianjun@tongji.edu.cn

Wen-Feng Zhu, born in 1976, is currently an professor at School of Mechanical Engineering, Tongji University, China. He received his $\mathrm{PhD}$ degree from Shanghai Jiaotong University, China, in 2005.His main research interests include advanced welding and connection technology, and mechanical behavior of materials and structures.

Tel: +86 13917863715; E-mail: zhuwenfeng@tongji.edu.cn

Yuan-Hui Li, born in 1995, is currently a master candidate at School of Mechanical Engineering, Tongji University, China.

E-mail: 1990492970@qq.com

\section{Corresponding author: Wen-Feng Zhu E-mail: zhuwenfeng@tongji.edu.cn}




\title{
ORIGINAL ARTICLE
}

\section{Numerical quantification model and experiment of external force on roller hemming of curved edge aluminium alloy with adhesive}

\author{
Jian-Jun $\mathrm{Li}^{1} \bullet$ Wen-Feng Zhu' ${ }^{1}$ Shun-Chao Wang ${ }^{1}$
}

Received August 12, 2021; revised xx xx, 2021; accepted xx xx, 2021

(C) Chinese Mechanical Engineering Society and Springer-Verlag Berlin Heidelberg 2017

\begin{abstract}
Accurate quantification of external force is the key to improve the high-precision hemming of autobody closure panels. However, the mechanism of external force on forming quality of complex contour sheet metal with adhesive is not clear subjected to geometric curvature and materials. In the present study, taking the curved edge aluminum sheet as the research object, SPH (smooth particle hydrodynamics) is introduced to simulate the viscous adhesive, and the SPH-FEM (Finite element method) coupling model of adhesive and panel considering the viscosity-pressure effect is established. The numerical simulation of the roller hemming process is carried out, then the validity and reliability are verified by real-time measurement of external force by triaxial force sensor. The multi-step forming process and the effect of external force on the roll in/out, surface wave and plastic strain of aluminum alloy sheet under the viscosity-pressure effect are studied, and the relationship between process parameters and external force is discussed. Results show that the coupling SPH-FEM model can well reflect the hemming process of curved edge structure. The normal force is about 2 3 times of the tangential force in the pre and final hemming process. Compared with the case without adhesive, the surface wave of flange part of the hemming with adhesive is slightly larger.The normal force and the tangential force increase about $90 \mathrm{~N}$ and $30 \mathrm{~N}$ respectively, when the height increases by $1 \mathrm{~cm}$. It provides an important basis for the accurate control of hemming trajectory and the improvement of manufacturing quality of autobody closure panels..
\end{abstract}

Keywords: External force • Forming - Adhesive - Simulation•

Wen-Feng Zhu

zhuwenfeng@tongji.edu.cn

1 School of Mechanical Engineering, Tongji University, Shanghai 201804, China
Aluminum alloy sheet $•$ Manufacturing quality

\section{Introduction}

Autobody closure panels, such as doors, hoods and decklids, have high requirements on the appearance accuracy of A-class surfaces, smooth contour and uniform clearance, which determines the static perception of the vehicle and affects the dynamic performance, reflecting the advanced level of manufacturing technology [1-2]. The assemblies are mainly composed of inner and outer panels, which are connected by hemming process [3-4]. Due to the complex mechanical interaction between workpiece and tool, high forming quality is related to many process parameters, of which the external force is the most important factor. The external force can not only reflect the forming quality on-line, but also provide the basis for the feedback and compensation of the monitoring system. Therefore, the accurate quantification of external force is of great importance for the complex contour panels with low density materials. With the acceleration of new model updating and shortening of trial-produce cycle, the relationship between external force and hemming quality has gradually become the focus of attention.

During the hemming process, the outer panel is prone to quality defects, such as roll in/out, wrinkles and cracks [5-7], subject to complex external load. The early research mainly focused on traditional hemming, such as die or tabletop hemming. Muderrisoglu et al. [8] studied the relationship between external load and punch stroke in different stages of flanging, pre hemming and final 
hemming. Le Maoût et al. [9] investigated effect of prestrain on load under horizontal and vertical displacement of the blade by finite element method. With the advantages of automation and flexibility, roller hemming gradually replaced the former, which uses an roller moving along contour path driven by an industrial robot [10-11]. Li et al. [12] predicted the change of external load with roller hemming time under cyclic stress Thuillier et al. [13] studied the variation of external load based on different constitutive models, and indicated that the irregular variation and vibration were related to element and friction. Eduardo et al. [14] proposed an off-line compensation method of external force to reduce the influence of dimension on forming quality. Drossel et al. [15] indicated the contact form between the roller and the workpiece was constantly changing, and finally affected the force and torque of robot. It can be seen that the geometric contour is an important factor affecting the external force and forming quality.

Due to its corrosion resistance and sealing function, the adhesive is sandwiched between the inner and outer panels during the hemming process. After the subsequent baking process, the adhesive is cured and tightly connected with the panels. Thus, it has replaced the traditional spot welding to avoid the dent of the outer panel, and become an important auxiliary connection means [16-17]. However, as a high viscosity fluid, the physical properties of adhesives are different from those of traditional metal materials, which makes numerical simulation and experimental quantification difficult. Svensson et al. [18] studied the hemming process with the adhesive for engine hood, and found that the results of simulation and experiment were quite different when simplified it. Li et al. [19] showed that the adhesive with a certain thickness had an effect on the roll in/out value. Burka et al. [20] indicated the viscous reaction was generated on the squeezing panel and affected by its composition. On the one hand, the flow of adhesive has an effect on sheet metal forming, and its effect can not be ignored. On the other hand, the obtained uniform thickness adhesive layer is conducive to the structural accuracy control. But defects, such as adhesive shortage and overflow, have adverse effects on subsequent subsequent baking process. Therefore, accurate forming of sheet metal with adhesive is a key problem in the quality control of the hemming process.

In summary, the basic research on the mechanical relationship between roller and work piece is still lacking. Affected by the coupling effect of dissimilar materials, the quantitative analysis is difficult to achieve. Thus it is urgent to establish a quantification model to reveal its mechanism and predicting forming quality, so as to provide basis for on-line monitoring, compensation and feedback of the system.

In this paper, the influence of external force on the forming quality of curved edge aluminum alloy sheet AA6016 is studied. SPH is introduced to simulate the viscous adhesive in flow state, and FEM is used to simulate the aluminum alloy sheet. The SPH-FEM coupled numerical model is developed, then the reliability of the simulation model is verified by the real-time detection of external force by triaxial mechanical sensor. The change of normal force and tangential force in different stages with adhesive and without adhesive is studied, and the effect on roll in/out and elastic-plastic strain is discussed. Finally, the relationship between process parameters and external force is studied.

\section{Modeling of hemming with adhesive based on SPH-FEM}

\subsection{SPH model of hemming adhesive}

After being rapid squeezed, the adhesive flows in the gap between the inner and outer panels, and there is no fixed flow field, which is difficult to quantify accurately. The traditional fluid-solid coupled method of Euler and FEM is difficult to track the free interface of adhesive during the roller hemming. Therefore, the mehsless SPH method is introduced to simulate the adhesive, which is suitable for solving large deformation and hydrodynamics problems [21]. Coupled with the FEM, it has been successfully applied in many fields due to its high efficiency [22]. Therefore, the same method can be used to solve the problem of roller hemming with adhesive.

SPH uses particles to represent liquids and assigns all physical and mathematical properties of fluids to SPH particles. SPH calculates the field variables of any point in the support domain by point interpolation approximation, as shown in Figure 1.

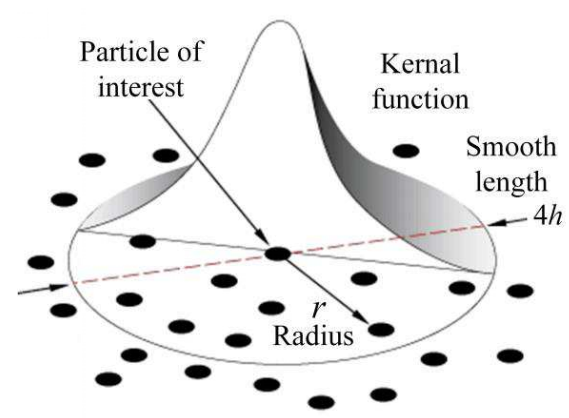

Figure 1 Smoothed particle and its support domain 
The smooth function $f\left(x_{\mathrm{i}}\right)$ and its derivatives in are expressed as follows [21]:

$$
\begin{gathered}
f\left(x_{i}\right)=\sum_{j=1}^{N} \frac{m_{j}}{\rho_{j}} f\left(x_{j}\right) \cdot W\left(\left|x_{i}-x_{j}, h\right|\right) \\
\nabla \cdot f\left(x_{i}\right)=-\sum_{j=1}^{N} \frac{m_{j}}{\rho_{j}} f\left(x_{j}\right) \cdot \nabla_{i} W\left(\left|x_{i}-x_{j}\right|, h\right)
\end{gathered}
$$

where $m_{j}$ and $\rho_{j}$ denotes mass and density respectively, $W$ is the smooth kernal function, and $h$ is smooth length. The most commonly used kernal function is cubic B-spline fuction, defined as:

$$
W\left(x-x^{\prime}, h\right)=C\left\{\begin{array}{cl}
1-1.5 u^{2}+0.75 u^{3} & 0 \leq u \leq 1 \\
0.25(2-u)^{3} & 1 \leq u \leq 2 \\
0 & u>2
\end{array}\right.
$$

where $C$ is a normalization. For three-dimensional problems, the value of constant $\mathrm{C}$ is $1 /\left(\pi h^{3}\right)$.

The mass conservation and momentum conservation functions in SPH form are described as follows [24]:

$$
\begin{gathered}
\frac{d \rho_{i}}{d t}=\sum_{j=1}^{N} m_{j}\left(v_{i}^{\beta}-v_{j}^{\beta}\right) \frac{\partial W_{i j}}{\partial x_{i}^{\beta}} \\
\frac{d v^{\alpha}}{d t}=\sum_{j=1}^{N} m_{j}\left(\frac{p_{i}}{\rho_{i}^{2}}+\frac{p_{j}}{\rho_{j}^{2}}\right) \nabla_{i} W_{i j}+ \\
\sum_{j}^{N} m_{j} \frac{\mu_{i}+\mu_{j}}{\rho_{i} \rho_{j}} v_{i j}^{\alpha}\left(\frac{1}{r_{i j}} \frac{\partial W_{i j}}{\partial r_{i j}}\right)+f_{s}
\end{gathered}
$$

Where $N$ represents the overall amount of particles in the influence region of particle $i$, and $p_{i}, \rho_{i}, \mu_{i}, v_{i}^{\alpha}$ denote the pressure, density, viscosity and speed. The vector $r_{i j}$ represents the gap, and $v_{i j}^{\alpha}$ represents the orientation. $W_{i j}$ denotes the smooth kernel function and $f_{\mathrm{s}}$ is the interfacial tension.

The total energy of the fluid remains unchanged, so it is still necessary to introduce the equation of state to decouple the two functions. The linear $U_{\mathrm{s}}-U_{\mathrm{p}}$ Hugoniot equation of state is expressed as [23]:

$$
p=\frac{p_{0} c_{0}^{2} \eta}{(1-s \eta)^{2}}\left(1-\frac{\Gamma_{0}}{2}\right)+\Gamma_{0} \phi \rho_{0} E_{m}
$$

Where $\eta$ donates the nominal volume compressive strain (equal to $\left.1-\rho_{0} / \rho\right), \Gamma_{0}$ is the material constant and $E_{\mathrm{m}}$ represents the initial value of unit internal energy. $\rho_{0}$ is the density, $c_{0}$ is the sound velocity, $U_{\mathrm{S}}$ is shock wave velocity, $U_{\mathrm{p}}$ is particle velocity. $s$ is the coefficient of linear relationship between $U_{\mathrm{s}}$ and $U_{\mathrm{p}}$.

The automotive hemming adhesive studied is a one component epoxy resin type adhesive. As a polymer fluid, it mainly shows tensile flow during the squeezing process, therefore the non-Newtonian characteristics of time dependence and shear rate are ignored. At normal temperature, it is simplified as high viscous Newtonian fluid, and its main parameters are shown in Table 1.

Table 1 Main performance parameters of the hemming adhesive

\begin{tabular}{lc}
\hline Parameters & Value \\
\hline Mass density & $1.45 \mathrm{~g} / \mathrm{cm}^{3}$ \\
Dynamic viscosity & $50 \mathrm{~Pa} \cdot \mathrm{s}$ \\
Curing conditions & $180^{\circ} \mathrm{C} / 30 \mathrm{~min}$ \\
Shear strength & $\geq 30 \mathrm{MPa}$ \\
Main components & Epoxy resin \\
\hline
\end{tabular}

\subsection{FEM model of metal sheet}

Sheet of AA6016 is selected as the research object, which is widely used in automobile outer panels due to high specific strength and formability. The mechanical properties of the material are obtained by a conventional servo-hydraulic testing machine. Compared with the rolling direction, uniaxial tensile tests are conducted along the directions of $0^{\circ}, 45^{\circ}$ and $90{ }^{\circ}$ with respect to the rolling direction, and the real stress-strain curve show isotropy characteristics. As the sheet metal is formed at room temperature and the hemming speed is uniform, the influence of strain rate and temperature on the material rate is ignored. Therefore, an elastic-plastic power law exponential model $\sigma_{p}=K\left(\varepsilon_{0}+\varepsilon_{p}\right)^{n}$ is considered in the numerical simulation, and the von Mises yield criterion is adopted. The major mechanical property parameters for FEM simulation are shown in Table 2.

Table 2 Major mechanical property parameters for FEM simulation

\begin{tabular}{lc}
\hline Mechanical Property & Material AA6016 \\
\hline Mass density & 2700 \\
Hardening index & 0.28 \\
Possion's ratio & 0.3 \\
Yield Strength & $124 \mathrm{MPa}$ \\
Tensile Strength & $229 \mathrm{MPa}$ \\
\hline
\end{tabular}

\subsection{SPH-FEM coupling algorithm}

FEM is a kind of Lagrangian method based on grid, 
which is good at calculating high-precision solid mechanics problems. SPH is a meshless Lagrangian particle method, and its accuracy is not affected by the degree of material deformation, so it is suitable for solving fluid dynamics problems. SPH and FEM have different application objects and fields, but they are complementary. The coupling of SPH and FEM can be realized by contact algorithm [21,24], as shown in Figure 2 . The contact force is added to SPH momentum equation and FEM equation in the form of external force:

$$
\begin{gathered}
\frac{\mathrm{d} v^{\alpha}}{\mathrm{d} t}=\sum_{j=1}^{N} m_{j}\left(\frac{p_{i}}{\rho_{i}^{2}}+\frac{p_{j}}{\rho_{j}^{2}}\right) \nabla_{i} W_{i j}+ \\
\sum_{j}^{N} m_{j} \frac{\mu_{i}+\mu_{j}}{\rho_{i} \rho_{j}} v_{i j}^{\alpha}\left(\frac{1}{r_{i j}} \frac{\partial W_{i j}}{\partial r_{i j}}\right)+f_{s}+f\left(x_{i}\right) \\
M \ddot{u}+C \dot{u}+K u=f\left(x_{i}\right)
\end{gathered}
$$

where $M$ is the structure mass matrix, $C$ is the structural damping matrix, $K$ is the structural stiffness matrix, and $u$ is the displacement at a certain time.

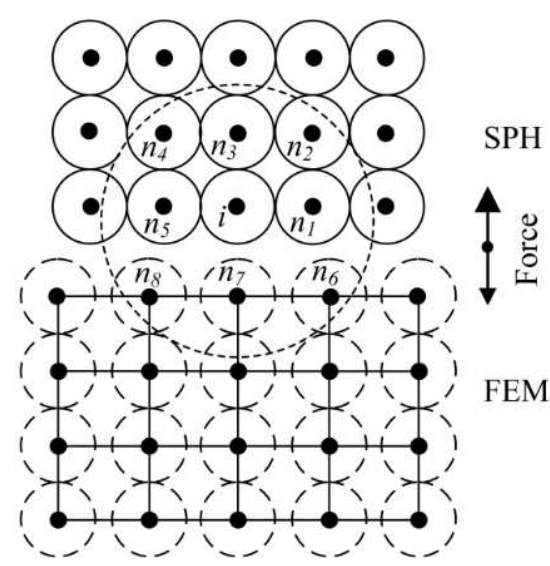

Figure 2 SPH-FEM element contact

The flow of coupling algorithm is shown in Figure 3. Considering the rheological properties of adhesive and the constitutive model of sheet metal, the coupled model with different geometric profiles and constraints is established. In each calculation time step, SPH particles and FEM elements pass node information to each other through contact algorithm, including mass, position and stress. The Leap-frog algorithm is used to solve the Navier-Stokes equations of SPH particles, and the finite element difference method is used to solve the explicit dynamic equations. Finally, the deformation of metal sheet and the distribution of adhesive layer in different stages are predicted. By changing the geometric and technological parameters, the mapping relationship between external force and forming quality is obtained.

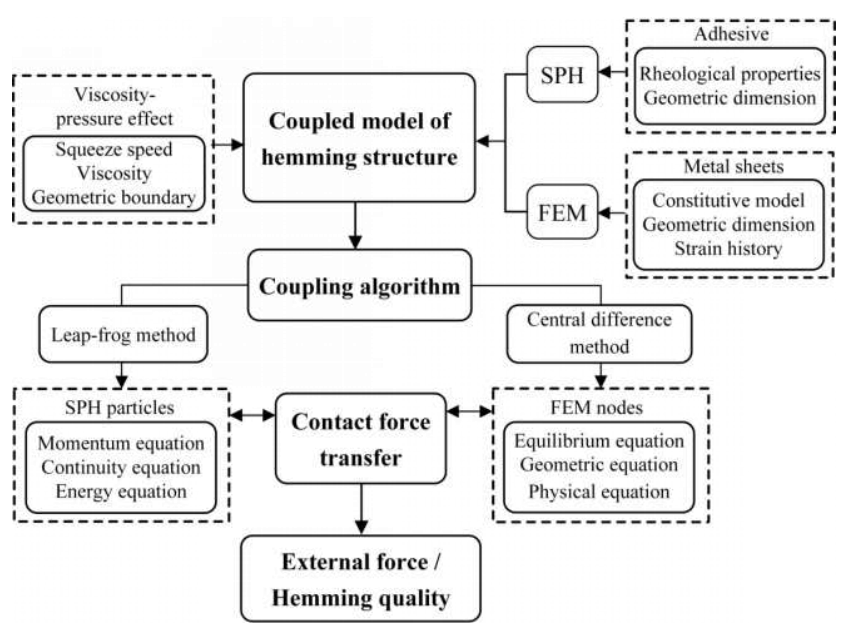

Figure 3 Flow chart of forming quality prediction based on SPH-FEM coupling model

In the numerical simulation model, the calculation accuracy of the solid element is higher than that of the shell in FEM, but the shell element can effectively reduce the calculation time. By increasing the number of thickness integration points, the forming accuracy of small radius bending can be improved.3,25 Therefore, the shell element with 5 integral points in thick direction is used to model the outer panel. In the bending area, the mesh size is $0.5 \mathrm{~mm}$, and it is sparse in other areas. The inner panel, punch, die and roller are set as rigid bodies. The adhesive is divided into SPH particles with the size of $0.1 \mathrm{~mm}$. In the flanged stage, the distance between the punch and die is 1.3 times of the panel thickness to avoid surface indentation and large springback. Since the surface of workpiece and roller is smooth, the friction coefficient is set to $0.15[5,6]$. In the multi-step simulation process, the method of mass scaling is adopted, and the computer multi-core calculation is carried out based on the commercial software.

\subsection{Experimental conditions and cases}

The experimental equipment includes industrial robot (KUKA KR600), triaxial force sensor (omega191 f/t), roller, modular fixture and platform, etc., as shown in Figure 4 . When the roller rotates, it can move vertically along the $\mathrm{y}$ direction and translate along the tangent direction $\mathrm{z}$ of the trajectory. The sensor is installed at the end of the robot arm, and the real-time data of the sensing signal is converted by the processor and transmitted to the 
computer. It can monitor the force in three directions at the same time.
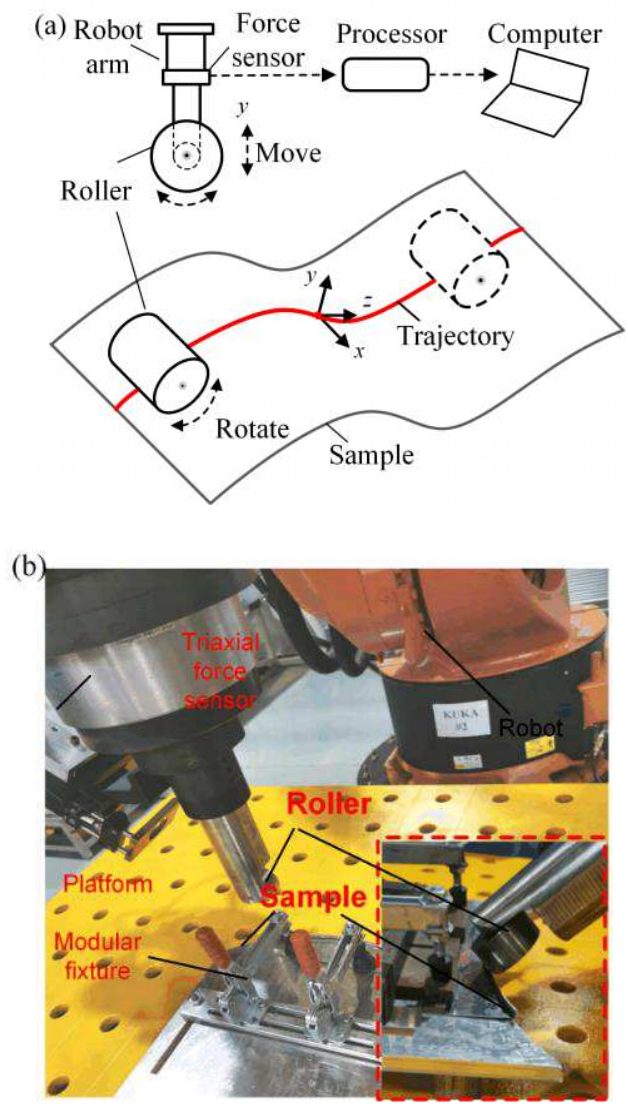

Figure 4 Roller hemming experiment. (a) Schematic diagram of external force monitoring; (b) Experimental device

The existence of curvature changes the forming boundary conditions, which increases the risk of defects and the difficulty of control. Therefore, samples with typical convex and concave edge profiles are selected, as shown in Figure 5. In order to quantify the parameters conveniently, different sections are divided on the curve sample, which are evenly distributed at equal angles, $S 1$, $S 2 \ldots$ and $S 13$.

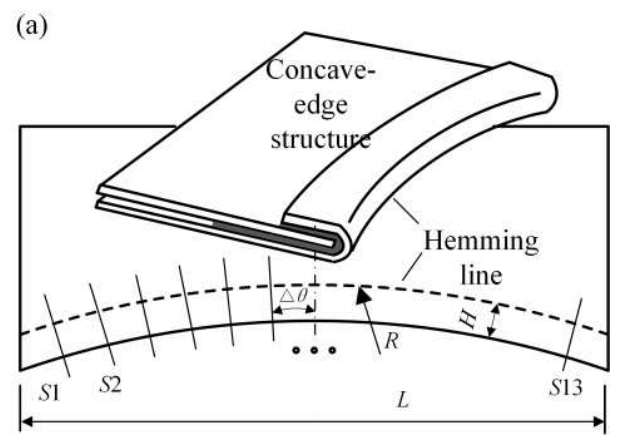

(b)

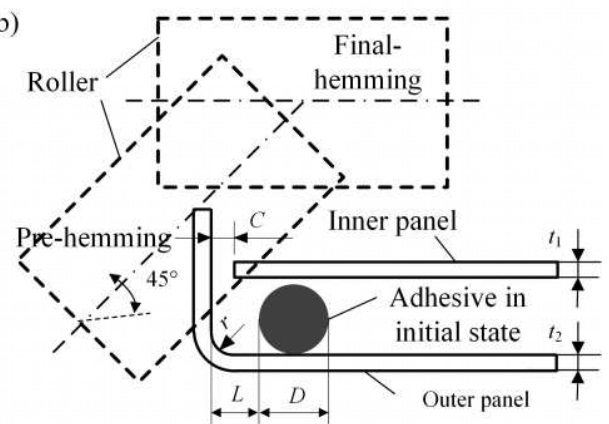

Figure 5 Schematic diagram of sample geometry. (a) Planar structure with concave edges; (b) Initial state of adhesive in the cross section

Different from the straight edge, the strain is easy to accumulate during the multi-step process, and obvious wrinkles and fracture appear in the final hemming. Research showed that the defect were related to the contour radius $R_{\mathrm{C}}$ and flanged height $L_{\mathrm{F}}^{3,4}$. Therefore, the typical structural dimensions are selected in order to ensure the final quality. The specific parameters are shown in Table 3. The roll in/out value of sample is measured by vernier caliper, three times each time, and the average value is made after experiment.

Table 3 Geometric parameters of samples $\mathrm{mm}$

\begin{tabular}{lc}
\hline Parameter names & Dimensions \\
\hline Radius of edge curve (Ra) & 240 \\
Distance $L$ & 2.2 \\
Distance $C$ & 1.2 \\
Sheet thickness $\left(t_{1} 、 t_{2}\right)$ & 0.8 \\
Flange height $(H)$ & 7.0 \\
Diameter of the adhesive $D$ & 3.2 \\
Inner flange radius $(r)$ & 0.8 \\
\hline
\end{tabular}

\section{Results and discussion}

\subsection{Comparison of simulation and experiment results}

As the strain history can affect the final forming quality, thus the flanging process is included (Figure 6a). It can be seen that the outer panel has entered plastic deformation, and the strain at the corner is relatively large while the flange has been in circumferential tensile state owing to the concave edge structure (Figure 6b). SPH particle is used to simulate the hemming adhesive, which was cylindrical and evenly distributed along the hoop direction (Figure 6c). After the inner panel is pressed vertically, the adhesive flows to the bending corner, and finally it reaches the predetermined thickness (Figure 6d). As the inter panel is simplified as a rigid body, there is no strain caused by the adhesive. During the pre hemming of fluid structure coupling interaction, there is a certain 
accumulation of adhesive at the flange (Figure 6e). The adhesive is extruded and flows upward, and finally realizes the distribution in the gap between the upper and lower panels during the final hemming (Figure 6h). It can be seen that the coupling model can well reflect the hemming process of curved edge structure.

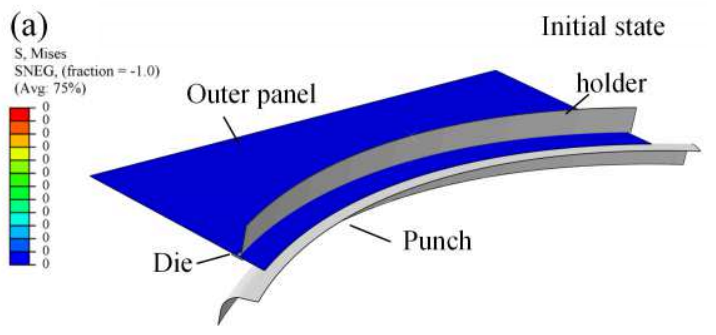

(b)

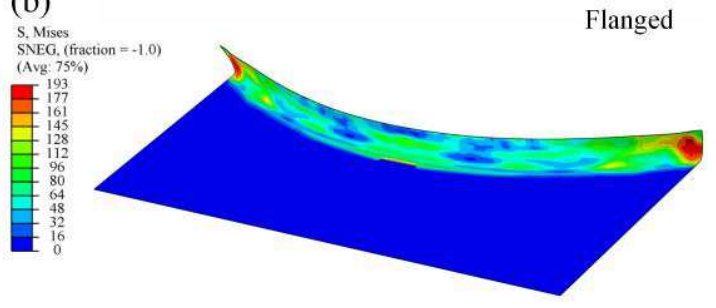

(c)

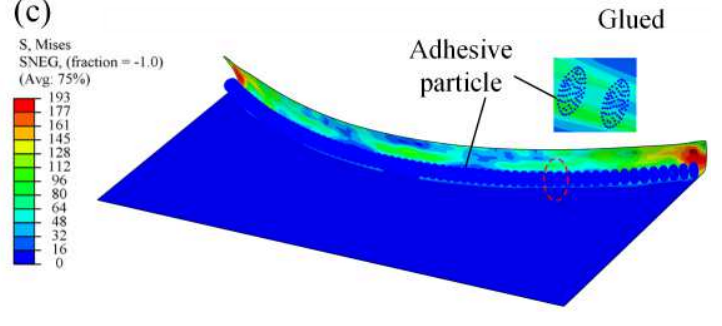

(d)

Compressed

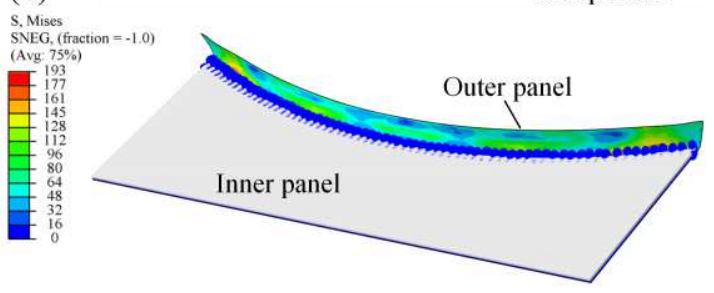

(e)

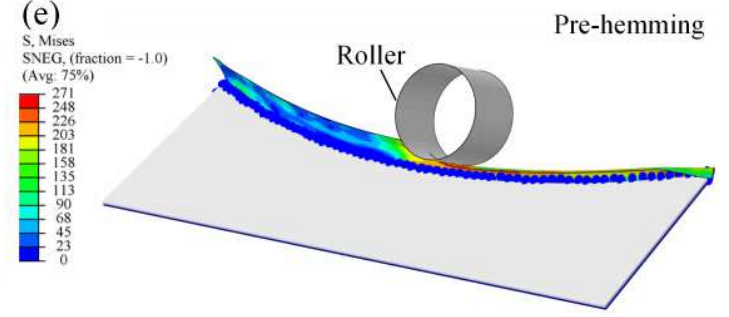

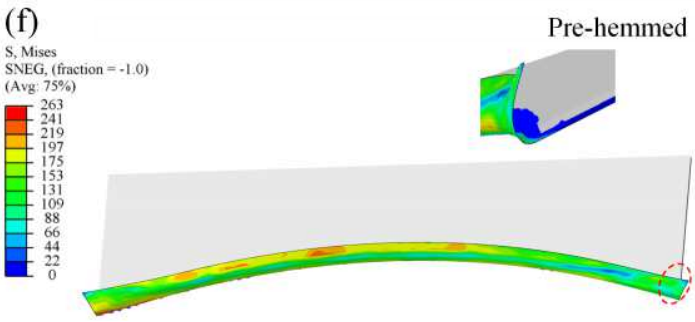

(g)

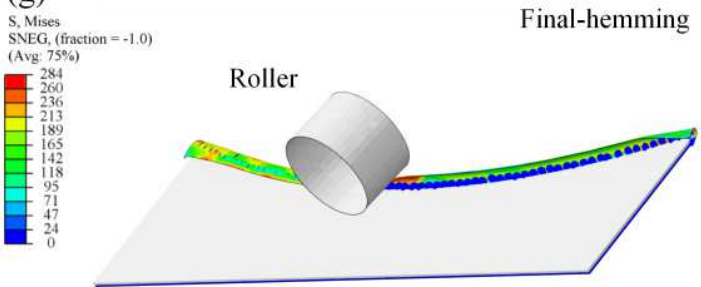

(h)

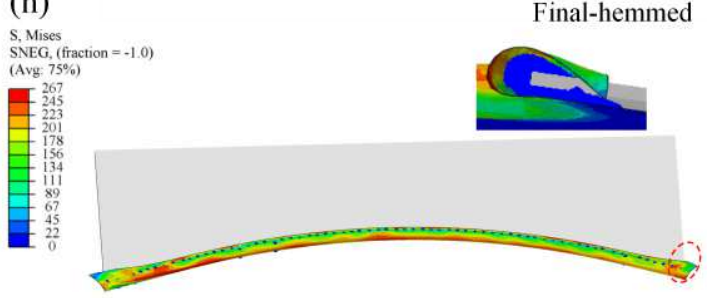

Figure 6 Roller hemming process of concave edge panels with adhesive.

The external force is divided into the normal force perpendicular to the workpiece and the tangential force moving along the tangential trajectory. Figure 7 shows evolution of the external force in the roller hemming process by experiment and simulation method. It can be seen that the external force based on SPH-FEM quantification model is in good agreement with the experimental measurement. In general, the normal force is greater than the tangential force and is the main action form. On the one hand, in the flanged area of sheet metal, sheet metal forming is a cycle hardening process. In the contact area with the roller, the strain of the panel is first stretched and then compressed [12]. In particular, under the viscosity-pressure effect of the adhesive, the external force obtained by simulation or experiment fluctuates in a small range. On the other hand, for the simulation calculation, the fluctuate also involves grid generation and iterative computation. Based on the principle of force equilibrium, the normal force on the roller is balanced with the bending opposing force on the outer panel. It can be seen that the normal force of the sample is greater in the final hemming than that in the pre hemming. This is 
because the larger the bending angle of the thin panel and the smaller the radius, the greater the reverse bending moment produced. The increase in the plastic deformation of the panel strengthens the material properties, resulting in an increase in the normal force in the final hemming stage. The normal force of the specimen without adhesive $(\mathrm{t}=2.4 \mathrm{~mm})$ increases mainly because the forming radius decreases and the plastic bending resistance increases.
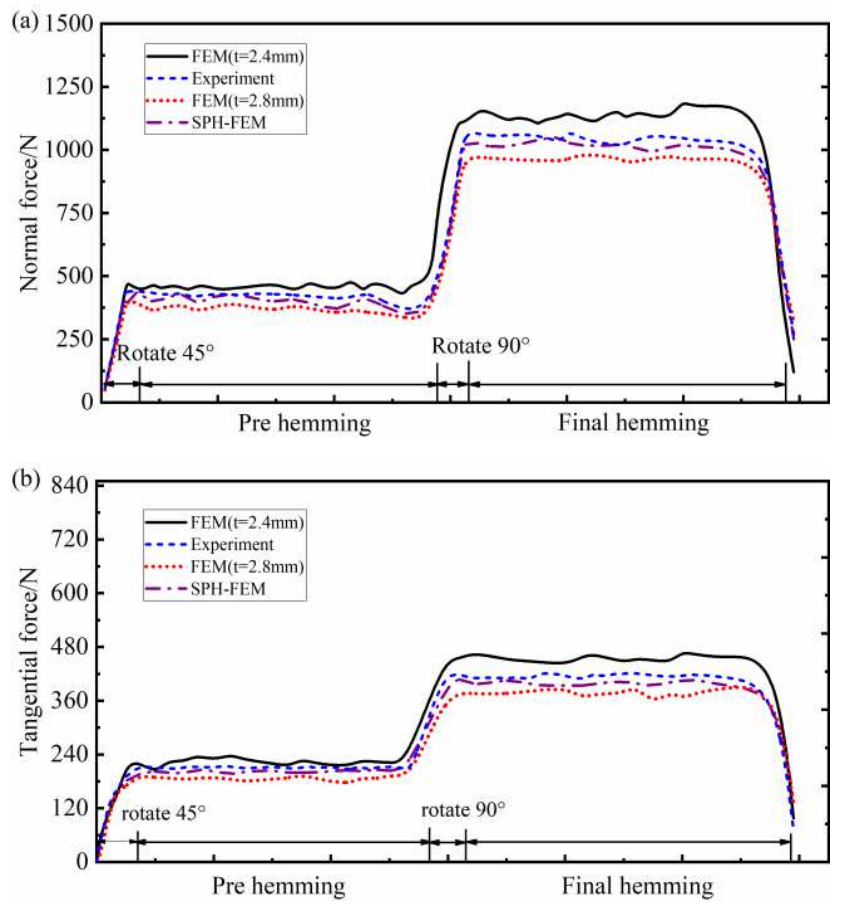

Figure 7 Evolution of external force on concave edge structure by experiment and simulation. (a) Normal force; (b) Tangential force

In general, the normal force is about 2 3 times of the tangential force in the two stages. According to the theory of hydrodynamic lubrication ${ }^{26}$, when the fluid is rapidly squeezed, the internal viscosity-pressure effect makes a reaction force on the solid boundaries. Therefore, compared with the sample without adhesive $(\mathrm{t}=2.8 \mathrm{~mm})$, the outer panel of the sample with adhesive is subjected to the non-linearly transient changing viscosity-pressure at the bending corner and flanged area, resulting in a relative increase in the normal force and the tangential force .

\subsection{Action mechanism of external force}

Roll in/out can affect the final assembly clearance between the panels and the automobile, which is an important forming quality index. Figure 8 shows the change of roll in/out in different stages. It can be seen that the roll in/out value of coupled SPH-FEM method is close to that of experiment and can reflect the change of forming quality. In general, the outer panel retracts inward after pre hemming and then expands outward after final hemming. The reason of expand outward is mainly related to the flanged area subjected to the circumferential tensile state. The roll in value at both ends is relatively large, which is related to the free boundary conditions. Compared with the samples without adhesive $(\mathrm{t}=2.8$ $\mathrm{mm})$, the roll in value decreased, which was mainly owing to the change affected by the viscosity-pressure effect. The main reason for the increase of roll in value of samples without adhesive $(\mathrm{t}=2.4 \mathrm{~mm})$ is the decrease of bending radius.
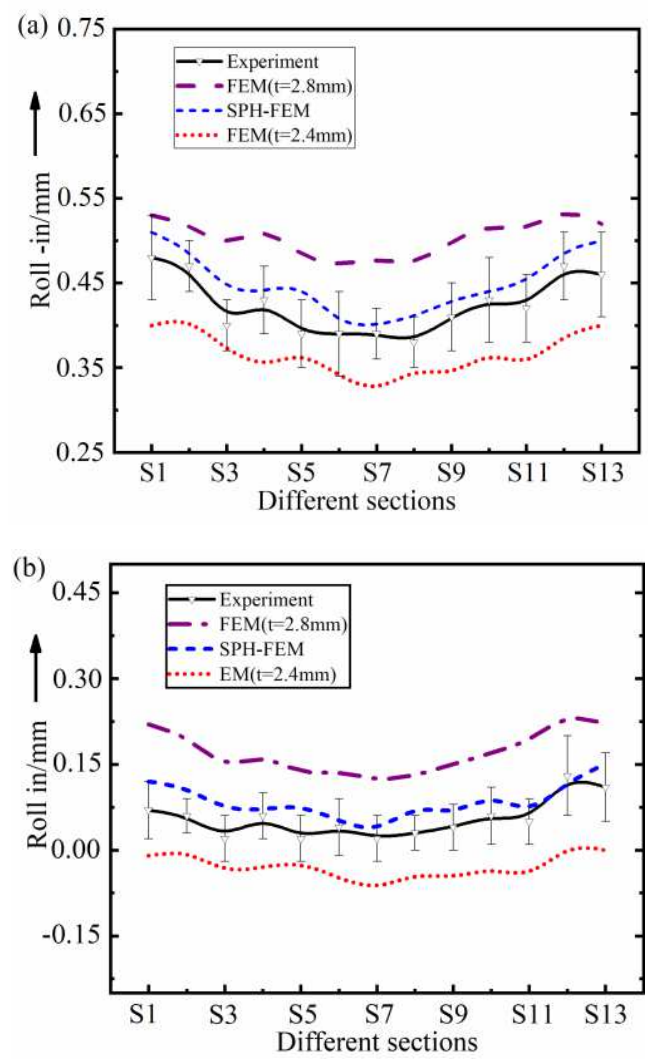

Figure 8 Changes of roll in/out at different sections. (a) Pre hemming; (b) Final hemming

Figure 9 shows the change of transient pressure and velocity of adhesive particles with time in the middle section. The adhesive is an incompressible fluid. After being squeezed, it flows rapidly in the narrow gap formed by the inner and outer panels, and the transient pressure appears inside. In the pre and final hemming stroke, there is a peak pressure, which produces a reaction force on the thin panel. The transient pressure of final rolling is slightly larger than that of pre hemming, which is related to the gap thickness and particle velocity. 


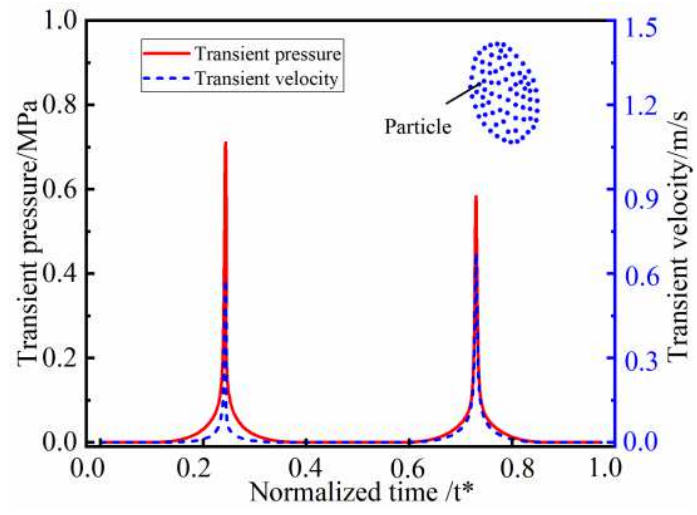

Figure 9 Transient pressure and velocity of SPH particles in the middle section

The surface flatness of the flanged part of the components not only affects the subsequent assembly quality, but also affects the distribution of the adhesive layer, which is an important forming index. Figure 10 shows the forming thickness and surface wave after final hemming. Compared with the case without adhesive, the simulation and experimental results show that the surface wave of hemming with adhesive is slightly larger. This is related to the rapid squeeze of the adhesive layer under the viscosity pressure effect. The schematic diagram of force interaction between roller and out panel is shown in Figure 11. Under the action of the roller, the contact area of the sheet is first extruded and then stretched. This results in cyclic tension compression strain in the sheet and wave wrinkling on the surface [11-12]. When there is no adhesive, the outer panel is in direct contact with the inner panel with high strength, so it is easy to flatten. Influence of adhesive layer on the lower part of panel. When there is adhesive, the transient instability of the panel is easy to occur, resulting in larger wave.

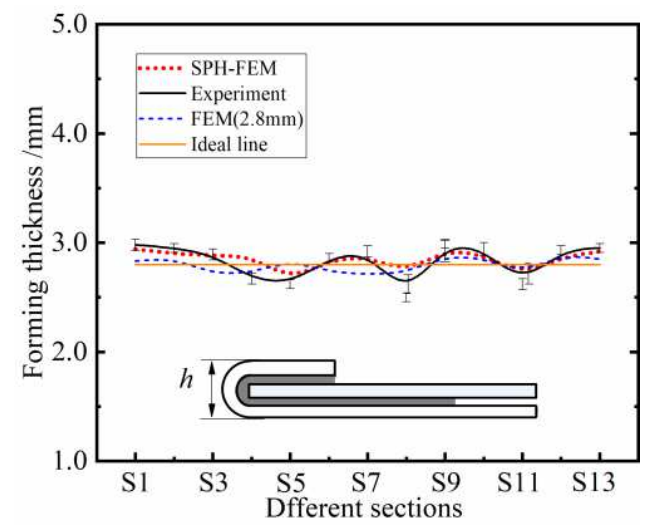

Figure 10 Forming thickness and surface wave after final hemming.

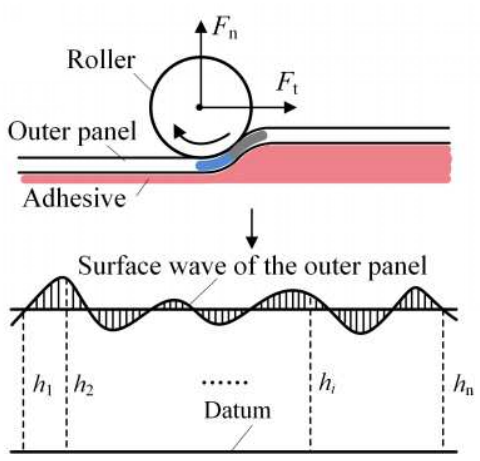

Figure 11 Schematic diagram of force interaction between roller and out panel

Figure 12 shows the elastic-plastic evolution of samples with and without hemming adhesive. From the experimental samples with $(\mathrm{t}=2.8 \mathrm{~mm})$ and without adhesive $(\mathrm{t}=2.4 \mathrm{~mm})$, It can be seen that the bending shape and surface texture have changed with different forming thickness.

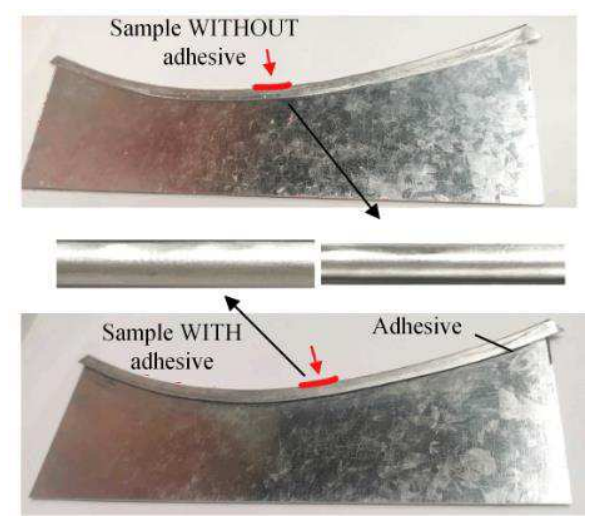

Figure 12 Experiment samples with and without adhesive after roller hemming.

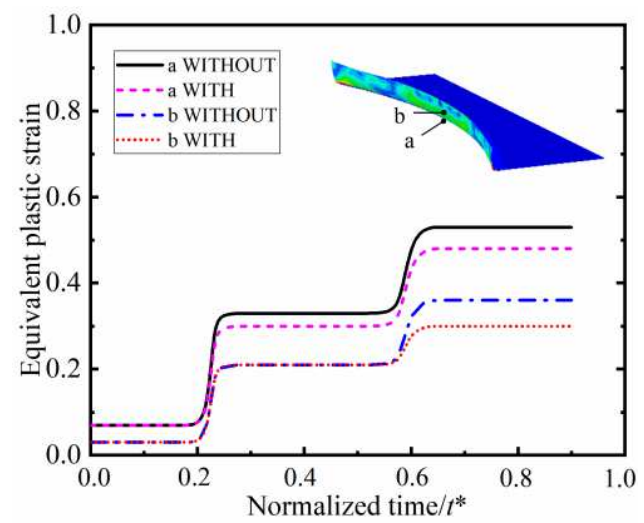

Figure 13 Evolution of equivalent plastic strain of samples with and without adhesive at different regions 
From the elastic-plastic value of different points in the middle section in Figure 13, it can be seen that after flanging, the plastic strain has been produced in the thin panel, and the calculation results considering historical strain are more accurate. The equivalent plastic strain with adhesive layer is getting smaller than that without adhesive layer on the whole. This is because, on the one hand, the panel is affected by the viscosity pressure effect. On the other hand, the contact action point between the roller and the outer panel changes. The change of boundary conditions leads to the change of the normal and tangential forces.

\subsection{Relationship between process parameters and}

\section{external force}

In the roller hemming process of closure panels with complex edge contour, the pose and stiffness of the roller system change all the time. When the system is abnormal, the fluctuation of external force may increase suddenly, leading to various quality defects. In order to realize on-line monitoring, feedback and compensation, it is necessary to establish the relationship between process parameters and external forces. For the convenience of intuitive comparison, the values of the exernal force on different sections are taken for average quantification.
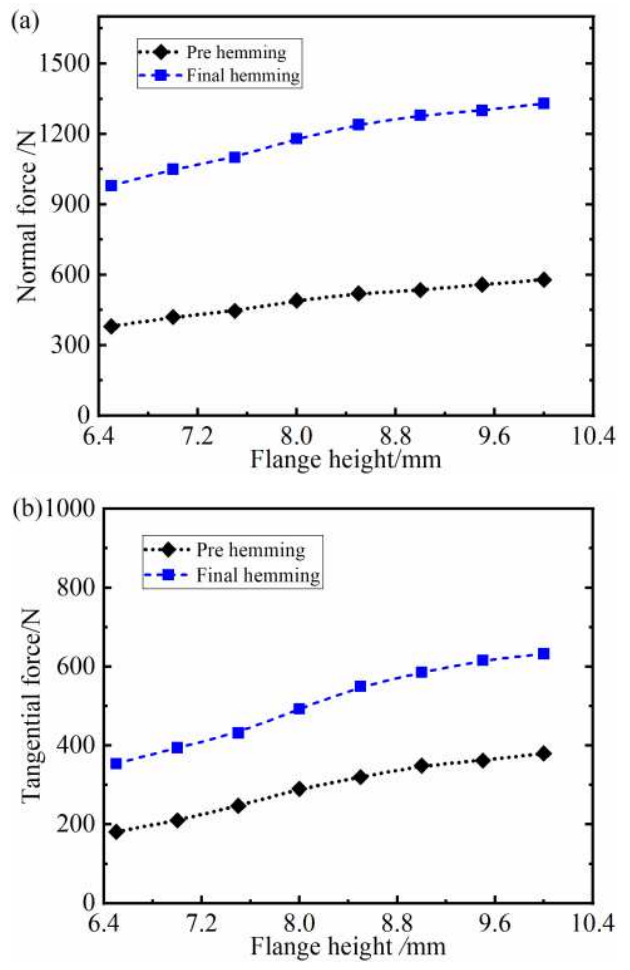

Figure 14 Evolution of external force with flanging height. (a) Normal force; (b) Tangential force
The flanging height is an important forming parameter, which directly reflects the connection quality and strength. Figure 14 shows the evolution of external force with flanging height, with other parameters unchanged. It can be seen that with the increase of the height, both the normal force and tangential force increase in two different stages. Especially in the final hemming stage, the normal force and the tangential force increase about $90 \mathrm{~N}$ and 30 $\mathrm{N}$ respectively, when the height increases by $1 \mathrm{~cm}$. The main reason is that with the increase of materials in the flanging area, the reverse bending moment and tangential resistance of the outer panel increase. When the height exceeds $9 \mathrm{~mm}$, the increasing rate of external force becomes slower, which is related to the form of concave flanging.
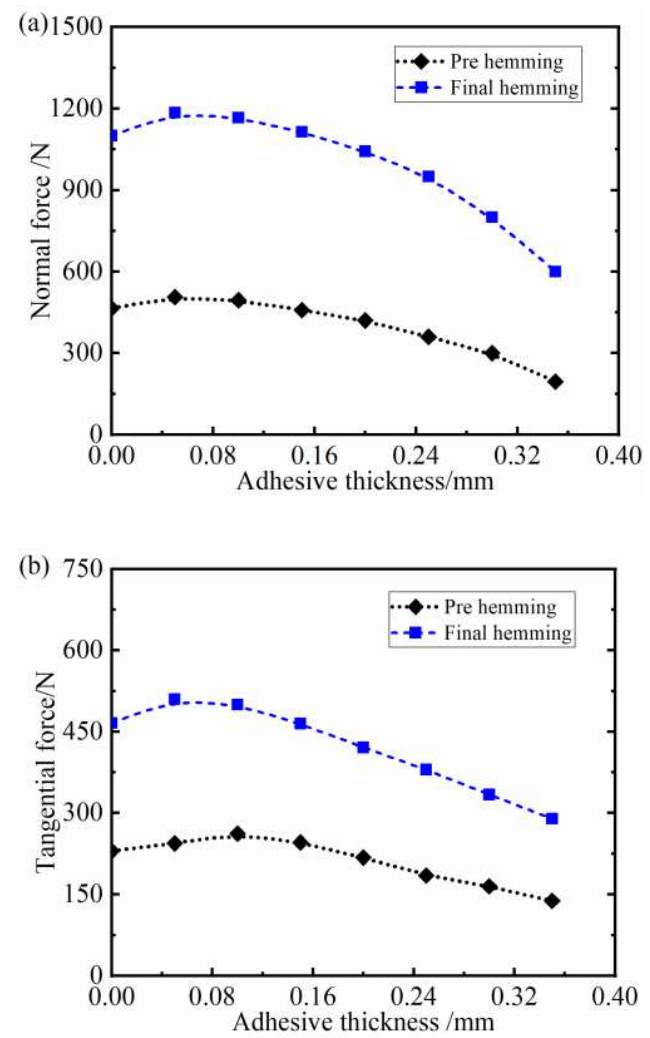

Figure 15 Evolution of external force with adhesive layer thickness

The accurate control of adhesive layer thickness not only affects the flow distribution of the adhesive, but also directly affects the total forming thickness of the assembly, which is another important process parameter. Figure 15 shows the evolution of external force with the adhesive layer thickness, with other parameters unchanged. With the increase of thickness, the external force first increases and then decreases. When the thickness of adhesive layer is close to $0.05 \mathrm{~mm}$, the external force is the largest. The 
smaller the extrusion thickness is, the more obvious the viscosity pressure effect is. With the increase of the thickness of the adhesive layer, the external force decreases, especially when the normal force exceeds 0.3 $\mathrm{mm}$. The main reason is that with the increase of the bending radius, the bending moment of the thin panel decreases, and the influence of the adhesive decreases obviously.
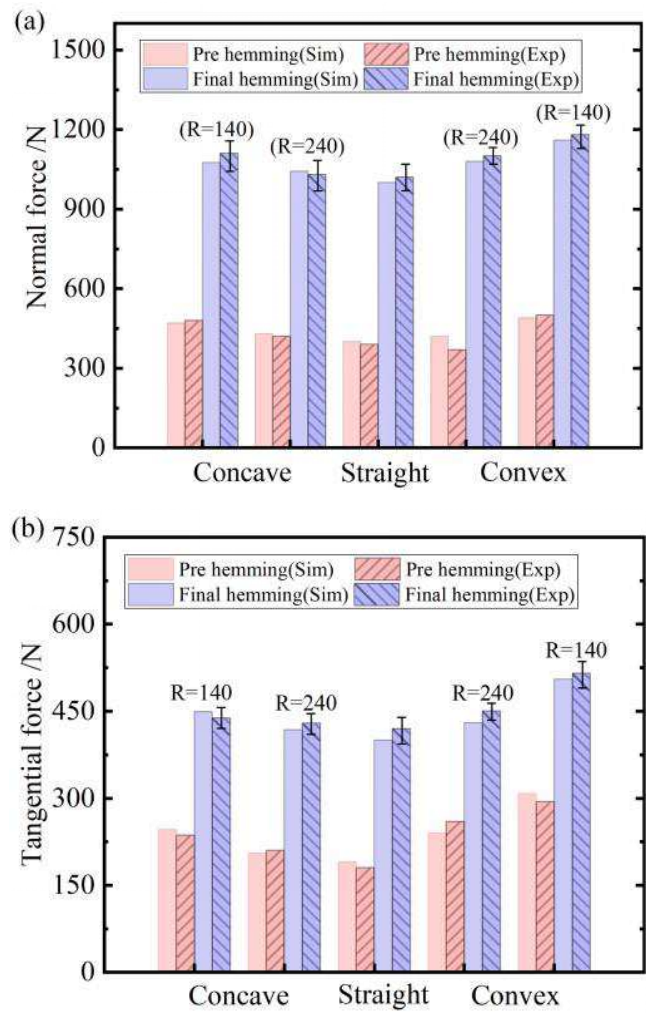

Figure 16 Influence of contour form and radius on external force

The variation of the geometric contour changes the squeezing boundary conditions and also causes the change of external forces. Figure 16 shows the effect of contour shape and radius on flanging force. In general, the external force of the curved edge sample is greater than that of the straight edge, which is related to the plastic strengthening of the flanged part with the curved edge. With the decrease of radius, the external force of the convex edge is slightly greater than that of concave edge. In the final hemming, the external force with small radius ( $\mathrm{R}=140 \mathrm{~mm}$ ) concave and convex edge is respectively increased by about $70 \mathrm{~N}$ and $100 \mathrm{~N}$, compared with the straight edge. This is because the material is easy to pile up on the convex edge structure subjected to circumferential load, so it needs to produce greater force when flattening to the process thickness.

\section{Conclusions}

The numerical quantification model of roller hemming of curved edge AA6016 aluminum alloy with adhesive is established, and the mechanism of external force on the the surface quality and gluing of adhesive is studied.Meanwhile, the relationship between process parameters and external force is studied. The main conclusions are as follows:

(1) The coupling SPH-FEM model can well reflect the hemming process of curved edge structure. The external force based on the numerical quantification model is verified by experiments, which provides an effective method to obtain the external force magnitude and direction.

(2) In general, the normal force is about 2 3 times of the tangential force in the pre and final hemming process. And the external force is greater in the final hemming than that in the pre hemming. This is because the larger the bending angle of the thin panel and the smaller the radius, the greater the reverse bending moment produced.

(3) The concave edge structure retracts inward after pre hemming, and expand outward after final hemming. Compared with the case without adhesive, the surface wave of flange part of the hemming with adhesive is slightly larger, and the equivalent plastic strain in the bending and flanging regions decreases, which is related to the viscosity-pressure effect.

(4) The normal force and the tangential force increase about $90 \mathrm{~N}$ and $30 \mathrm{~N}$ respectively, when the height increases by $1 \mathrm{~cm}$. With the increase of the thickness of the adhesive layer, the external force decreases, especially when the normal force exceeds $0.3 \mathrm{~mm}$. The external force with small radius $(\mathrm{R}=140 \mathrm{~mm})$ concave and convex edge is respectively increased by about 70 $\mathrm{N}$ and $100 \mathrm{~N}$, compared with the straight edge.

\section{Declaration}

\section{Acknowledgements}

The authors sincerely thanks to the team members in our research group for their critical discussion and reading during manuscript preparation. The authors sincerely thanks to Professor Jun-Ying Min of Tongji University for providing the experimental platform.

\section{Funding}

Supported by the NSFC-China (National Natural Science 
Foundation of China) Project (No.51975416 and No.51275359).

\section{Availability of data and materials}

The datasets supporting the conclusions of this article are included within the article.

\section{Authors' contributions}

The author' contributions are as follows: Jian-Jun Li completed the simulation and experiment analysis, and wrote the manuscript. Wen-Feng Zhu was in charge of framework and research methods of the whole manuscript. Shun-Chao Wang assisted with sampling and experiments.

\section{Competing interests}

The authors declare no competing financial interests.

\section{References}

[1] A Andersson. Evaluation and visualisation of surface defects on auto-body panels. Journal of Materials Processing Technology, 2009, 209: 821-37.

[2] P Martensson, $\mathrm{D}$ Zenkert, $\mathrm{M}$ Akermo. Method for cost and weight-efficient material diversity and partitioning of a carbon fibre composite body structure. Proceedings of The Institution of Mechanical Engineers Part D-Journal of Automobile Engineering, 2016: 0954407015578037.

[3] Gürgen, Selim. A parametric investigation of roller hemming operation on a curved edge part. Archives of Civil and Mechanical Engineering, 2018: 19(1): 11-19.

[4] G Lin, J Li, S Hu, et al. A computational response surface study of three-dimensional aluminum hemming using solid-to-shell mapping. Journal of Engineering Materials and Technology-Transactions of The ASME, 2007: 129(2): 360-368.

[5] H Livatyali, T Laxhuber, T Altan. Improvement of hem quality by optimizing flanging and pre-hemming operations using computer aided die design. Journal of Materials Processing Technology, 2000, 98: 41-52.

[6] G Zhang, X Wu, S J Hu. A study on fundamental mechanisms of warp and recoil in hemming. Journal of Engineering Materials and Technology-Transactions of The ASME, 2003, 123(4): 436-441.

[7] $\mathrm{G}$ Wang, $\mathrm{Z} \mathrm{Gu}, \mathrm{H} \mathrm{Xu}$, et al. Numerical analysis and experimental investigation of surface defects in die hemming process. International Journal of Material Forming, 2019, 13: 91-102.

[8] A Muderrisoglu, M Murata, M A Ahmetoglu, et al. Bending, flanging and hemming of sheet-experimental study. Journal of Materials Processing Technology, 1996, 59(1-2): 10-17.

[9] N Le Maoût, P YManach, S Thuillier. Influence of prestrain on the numerical simulation of the roller hemming process. Journal of Materials Processing Technology, 2012, 212(2): 450-457.

[10] N Le Maoût, S Thuillier, P Y Manach. Classical and roll-hemming processes of pre-strained metallic sheets. Experimental mechanics, 2010, 50(7): 1087-1097.

[11] X Hu, Z Q Lin, S Li, et al. Fracture limit prediction for roller hemming of aluminum alloy sheet. Materials \& Design, 2011, 31(3): 1410-1416.

[12] $\mathrm{S} \mathrm{Li}, \mathrm{X} \mathrm{Hu}, \mathrm{Y}$ Zhao, et al. Cyclic hardening behavior of roller hemming in the case of aluminum alloy sheets. Materials \& Design, 2011, 32(4), 2308-2316.

[13] S Thuillier, N Le Maoût, P Y Manach. Numerical simulation of the roller hemming process. Journal of Materials Processing Technology, 2008, 198(1-3): 226-233.

[14] E Eduardo, C Giuseppe, C Marco, et al. A Dynamic compensation for roll hemming process. IEEE Access, 2018, 6: 18264-18275.

[15] W G Drossel, M Pfeifer, M Findeisen, et al. The influence of the robot's stiffness on roller hemming processes. ISR/Robotik, 41st International Symposium on Robotics, Munich, Germany, june, 2014: 531-538.

[16] H S Cho, J U Cho. The characteristics of shear adhesive interface fracture of aluminum foam DCB bonded using a single-lap method. International Journal of Precision Engineering and Manufacturing, 2014, 15(7): 1345-1350.

[17] M F S F Moura, J P M Gonçalves, J AG Chousal, et al. Cohesive and continuum mixed-mode damage models applied to the simulation of the mechanical behaviour of bonded joints. International Journal of Adhesion and Adhesives, 2008, 28(8): 419-426.

[18] M Svensson, K Mattiasson. Three-dimensional simulation of hemming with the explicit FE-Method. Journal of Materials Processing Technology, 2002, 128(1-3): 142-154.

[19] J Li, W Zhu. Numerical simulation of the roller hemming process based on pressure-viscosity effect. International Journal of Advanced Manufacturing Technology, 2019, 105: 1023-1039.

[20] P Burka, X Liu, M C Thompson . Modelling of adhesive bonding for aircraft structures applying the insertion squeeze flow method. Composites Part B-Engineering, 2013, 50(7): 247-252.

[21] E Kara, A Kurşun, M R Haboğlu, et al. Fatigue behavior of adhesively bonded glass fiber reinforced plastic composites with different overlap lengths. Proceedings of The Institution of Mechanical Engineers Part C-Journal of Mechanical Engineering Science, 2015, 229(7): 1292-1299.

[22] Y Lin, L Yang, S Liu, et al. Virtual simulation technology based research on subsoiling process.Proceedings of The Institution of Mechanical Engineers Part C-Journal of Mechanical Engineering Science, 2018, 233(5): 1989-1996.

[23] K Gong, S Shao, H Liu, et al. Two-phase SPH simulation of fluid-structure interactions. Journal of Fluids and Structures, 2016, 65(8): 155-179.

[24] M Hallajisany, J Zamani, J A Vitoria. Numerical and theoretical determination of various materials Hugoniot relations based on the equation of state in high-temperature shock loading. High Pressure Research, 2019, 39(4): 666-690.

[25] V Abbas, K Morteza. Comparison of the numerical and experimental results of the sheet metal flange forming based on shell-elements types. International Journal of Precision Engineering and Manufacturing, 2011; 12(5): 857-863.

[26] Y Y Cho, T W Kim. Development of algorithm for 3D mixed Elasto-Hydrodynamic lubrication analysis. International Journal of Precision Engineering and Manufacturing, 2011, 12(6): 1065-1070.

\section{Biographical notes}

Jian-Jun Li, born in 1984, is currently a PhD candidate at School of Mechanical Engineering, Tongji University, China. He received his master degree from Shandong University, China, in 2011. His research interests include lightweight manufacturing of car body, material forming technology, mechanical behavior of materials and structures. 
Tel: +86 18953185993; E-mail: lijianjun@tongji.edu.cn

Wen-Feng Zhu, born in 1976, is currently an professor at School of Mechanical Engineering, Tongji University, China. He received his $\mathrm{PhD}$ degree from Shanghai Jiaotong University, China, in 2005.His main research interests include advanced welding and connection technology, and mechanical behavior of materials and structures.

Tel: +86 13917863715; E-mail: zhuwenfeng@ tongji.edu.cn

Shun-Chao Wang, born in 1996, is currently a master candidate at School of Mechanical Engineering, Tongji University, China.

E-mail: 2032760@qq.com 